\title{
Rationale for current drug treatment
}

\author{
F. BURKART
}

University Clinics, Basel, Switzerland

\begin{abstract}
KEY WORDS: Heart failure, symptoms of heart failure, medical treatment, prognosis.
In the initial phase of heart failure, cardiac output and pressure are maintained by increasing sympathetic drive and by cell hypertrophy. Elevated end-diastolic volume, a reduced ejection fraction and a higher filling pressure also occur. Only in more severe heart failure, when cardiac output cannot be maintained, do symptoms appear which may vary between congestion, exercise intolerance, left ventricular dysfunction, arrhythmias or a combination of each.

Drug treatment has principally two aims: first, to improve symptoms, second, to influence prognosis, which is very poor in advanced heart failure. Symptom improvement will also depend on whether the condition is acute, subacute or chronic heart failure. In the acute situation diuretics are normally the first choice of treatment, whereas in chronic heart failure the $A C E$ inhibitors have proved themselves to be the drugs which most improve prognosis. The role of digitalis, still frequently used, remains unclear, and its importance will be revealed when the results of ongoing studies are announced.
\end{abstract}

When contractility decreases or some myocardial fibres are lost due to necrosis, the organism has to compensate in order to maintain a normal circulation. In this initial phase of heart failure, metabolic demands and necessary perfusion pressure are maintained by the heart and circulation by increasing sympathetic drive and cell hypertrophy. Catecholamines lead to an increase in heart rate, by stimulation of renin-angiotensin, to sodium retention and thus to volume elevation and finally to vasoconstriction of the smaller vessels. When the diastolic volume of the ventricles increases, the contractile force of muscle fibres is enhanced according to Frank-Starling's law. In an early phase of heart failure we have, therefore, normal cardiac output at rest and under moderate exercise, but with a slightly increased heart rate, a higher than normal end-diastolic volume and therefore reduced ejection fraction, and an elevated filling pressure. In this situation, the patient has very few, if any, symptoms and only if effort tolerance is measured is a reduction in the maximal work load and in oxygen uptake found.

In more severe heart failure, normal cardiac output cannot be maintained and in order to guarantee an acceptable perfusion pressure, especially for the brain, the kidneys, and the heart, peripheral vasoconstriction occurs. With this elevated resistance, pressure can be maintained at an acceptable level, but at the cost of an increased work load on the failing heart. Volume retention and left ventricular end-diastolic pressure increases, leading finally to pulmonary congestion and, if the right heart also fails, to peripheral oedema. A low cardiac output also results in a reduced perfusion, not only of the skin and the intestines but also of the kidneys, leading to pre-renal failure, and to exercise intolerance of the muscles. Dilation of the heart, as well as hypertrophy, enhance ventricular ectopic activity which may become the dominant symptom. Figure 1

Correspondence. Felix Burkart, MD, FESC, Professor of Cardiology, Chief, Division of Cardiology, Unversity Clinies, Kantonsspital Basel, $\mathrm{CH}-4031$ Basel, Switzerland. demonstrates the different states that patients with the clinical syndrome of heart failure may show.

Drug treatment has principally two aims: first, to improve symptoms and with that quality of life; secondly, to improve prognosis, which is very poor once severe symptoms are present. From Figure 1 it becomes evident that drug treatment to improve symptoms depends on the clinical picture of the patient. This therapy may vary from that used to improve prognosis. In addition, drugs have different aims in the acute as compared to the chronic situation.

In acute heart failure, improvement in haemodynamics is the most important issue. Diuretics and vasodilators lower diastolic volume and pressure, improving dyspnoea and peripheral oedema. In patients with acute breathlessness, diuretics are very often the drug of first choice Comparative studies, however, have shown that with vasodilators a similar reduction of preload can be reached with less depression of cardiac output, which often occurs under diuretic treatment alone $\mathrm{e}^{[1]}$.

In the subacute situation, it is important to increase exercise tolerance by improving cardiac output, and here it seems that digitalis is still an important drug. Phosphodiesterase inhibitors, on the other hand, despite their beneficial haemodynamic effect, are no longer used due to their deleterious effect on prognosis. Here, improvement by drug treatment should be measured by effort tolerance tests, by measuring maximal oxygen uptake, and perhaps by questionnaires about quality of life.

In the chronic situation, improvement of left ventricular function, of neurohormonal status and of prognosis becomes the most important goal. It seems that the drugs of choice are ACE inhibitors. In the Veterans Administration Heart Failure Trial II (V-HeFT II) study Cohn and co-workers could demonstrate that the change in peak oxygen consumption and in ejection fraction was more pronounced with a combination of hydralazine and isosorbide dinitrate, whereas prognosis in these same patients was better in the enalapril-treated group ${ }^{[2]}$. This 


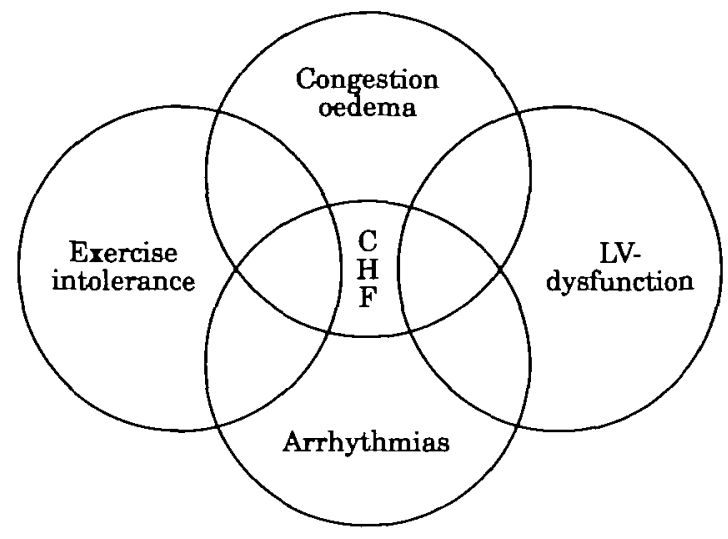

Figure 1 Clinical syndrome of heart failure.

study demonstrates that there may well be a difference in improving symptoms compared to prognosis. Large trials in recent years with $\mathrm{ACE}$ inhibitors showed that not only patients with severe symptoms (New York Heart Association classification class III to IV) have a better prognosis with this treatment $t^{[3]}$. Patients with asymptomatic left ventricular dysfunction demonstrated a reduced morbidity and mortality compared to those who did not receive this drug ${ }^{[45]}$. In a substudy of the Studies of Left Ventricular Function (SOLVD) trial it could be demonstrated that under ACE inhibition, ventricular enlargement could be reversed for the first 2 years after start of treatment. In the third year, the control end-diastolic volume was again reached, but the placebo group had demonstrated a continuous enlargement of the ventricle at the same time ${ }^{[0]}$. These data suggest that it is more important to decrease end-diastolic volume than improve cardiac contractility.

It is now generally accepted that the presence of complex arrhythmias worsens prognosis of patients in heart failure. Despite the fact that with class III antiarrhythmic drugs we may improve prognosis after myocardial infarction $^{[7]}$ this does not seem to be possible when left ventricular function is severely depressed ${ }^{[8,9]}$.
For the individual patient, therefore, we have to consider all these different facts. In severe left ventricular dysfunction, an ACE inhibitor is needed to improve prognosis. In patients with symptoms of congestion a diuretic has to be added and in those where effort intolerance dominates, a combination of ACE inhibitor with digitalis and/or vasodilators may be preferable.

\section{References}

[1] Nelson GI, Silke B, Ahuya RC et al. Hemodynamic advantages of isosorbid-dinitrate over frusemide in acute heart failure following myocardial infarction. Lancet $1983 ; 1$; 730-3.

[2] Cohn JN, Johnson G, Ziesche S et al. A comparison of enalapril with hydralazine isosorbide dinitrate in the treatment of chronic congestive heart failure. N Engl J Med 1991; 325: 303-10.

[3] The CONSENSUS trial study group. Effects of enalapril on mortality in severe congestive heart failure. Results of the cooperative north Scandinavian enalapril survival study. $\mathbf{N}$ Engl J Med 1987; 316: 1429-35.

[4] Pfeffer MA, Braunwald E, Moyé LA et al. Effect of captopril on mortality and morbidity in patients with left ventricular dysfunction after myocardial infarction. N Engl J Med 1992; 327: 669-77.

[5] The SOLVD investigators. Effect of enalapril on survival in patients with reduced left venricular ejection fractions and congestive heart failure. N Engl J Med 1991; 325: 293-302.

[6] Konstam MA, Rousseau MF, Kronenberg MW et al. Effects of the angiotensin converting enzyme inhibitor enalapril on the long-term progression of left ventricular dysfunction in patients with heart failure. Circulation 1992; 86: 431-8.

[7] Burkart F, Pfisterer M, Kiowski W et al. Effect of antiarrhythmic therapy on mortality in survivors of myocardial infarction with asymptomatic complex ventricular arrhythmias (BASIS). J Am Coll Cardiol 1990; 16: 1711-18.

[8] Pfisterer M, Kiowski W, Burckhardt D et al. Beneficial effect of amiodarone on cardiac mortality in patients with asymptomatic complex ventricular arrhythmias after acute myocardial infarction and preserved but not impaired left ventricular function. Am J Cardiol 1992; 69: 1399-1402.

[9] Nicklas JM, McKenna WJ, Stewart RA et al. Prospective, double-blind, placebo-controlled trial of low-dose amiodarone in patients with severe heart failure and asymptomatic frequent ventricular ectopy. Am Heart J 1991; 122: 1016-21. 